\title{
MAGNETIC FLUIDS: BIOMEDICAL APPLICATIONS AND MAGNETIC FRACTIONATION
}

\author{
THOMAS RHEINLÄNDER ${ }^{\mathrm{a}, *}$, RÓMAN KÖTITZ ${ }^{\mathrm{a}}$, \\ WERNER WEITSCHIES ${ }^{\mathrm{a}, \mathrm{b}}$ and WOLFHARD SEMMLER ${ }^{\mathrm{a}, \mathrm{c}}$ \\ a Institut för Diagnostikforschung $\mathrm{GmbH}$, Spandauer Damm 130, \\ D-14050 Berlin, Germany; ${ }^{\mathrm{b}}$ Ernst-Moritz-Arndt-Universität \\ Greifswald, Institut für Pharmazie, Friedrich-Ludwig-Jahn-Straße 17, \\ D-17487 Greifswald, Germany; ' Deutsches Krebsforschungszentrum, \\ Abteilung Biophysik und medizinische Strahlenphysik, \\ D-69120 Heidelberg, Germany
}

(Received 18 April 2000; In final form 22 May 2000)

In addition to engineering applications, magnetic fluids containing magnetic nanoparticles are being increasingly applied to biomedical purposes. Besides the well established use of magnetic particles for biological separation or as contrast agents for magnetic resonance imaging, magnetic particles are also being tested for the inductive heat treatment of tumors or as markers for the quantification of biologically active substances.

The properties of magnetic nanoparticles usually exhibit a broad distribution, so in many cases upon application only a small fraction of the particles contribute fully to the desired magnetic effect. Therefore, magnetic fluids have to be optimized by fractionation techniques. This is preferentially achieved by methods that separate magnetic nanoparticles in accordance with their magnetic properties. Hence, a magnetic technique has been developed for the fractionation of magnetic fluids. Two different magnetic fluids were fractionated by this method. The fractions obtained and the original samples were characterized with respect to their magnetic properties as well as their particle sizes. They were investigated not only in terms of their magnetization curves but also in respect to biomedical applications. The magnetic fractions show clearly improved magnetic properties compared to the original samples and are therefore especially suited for distinct applications. Furthermore, the results indicate that the magnetic method fractionates the particles in accordance with their magnetic moment and has a good reproducibility.

Keywords: Magnetic fluids; Magnetic nanoparticles; Magnetic fractionation; Magnetic resonance; Magnetic relaxation; Hyperthermia

*Address for correspondence: Mifa AG, Rheinstrasse 99, CH-4402 Frenkendorf, Switzerland. e-mail: th.rheinlaender@gmx.net 


\section{INTRODUCTION}

Magnetic fluids, also called ferrofluids, are usually stable suspensions of magnetic nanoparticles. These are single-domain particles due to their small size and consist of ferro- or ferrimagnetic material, mostly iron oxide. The magnetic particles are often coated with polymers, surfactants or charged compounds in order to stabilize them by steric and/or electrostatic repulsion. Magnetic fluids are widely applied in technical fields, e.g., for material separation, magnetic domain detection, pressure-tight rotary-shaft sealing or as damping and cooling agents for loudspeakers [1].

Several biomedical applications of magnetic nanoparticles are presented below. All of these applications utilize magnetic properties of nanoparticles such as their magnetic moment, which depends on their magnetic core size. In common magnetic fluids these parameters usually exhibit a broad distribution. Thus, in many cases only a small portion of particles contributes to the desired magnetic effect. The relative amount of these particles can be increased by the separation of magnetic fluids. Separation divides a material into two fractions only in each step, whereas fractionation parts a source into several fractions. Only in the case of fractionation the middle fractions are cut off bilaterally. Furthermore, fractionation is not only of preparative interest but can also be used for analytical purposes since it permits a more comprehensive description of polydispersed systems.

Common methods currently used for the fractionation of magnetic fluids are, for example, centrifugation [2] or size-exclusion chromatography [3]. All these methods are based on non-magnetic properties like density, size or stability. Preference should be given to partitions conforming to the properties of interest, which in this case are the magnetic properties. So far magnetic methods have been used for the separation of magnetic nanoparticles, e.g., for the removal of aggregates (magnetic filtration) [4] or of weakly magnetic or nonmagnetic material like synthesis by-products $[5,6]$. The highgradient magnetic separation required is possible through magnetized filtration elements as well as ones carrying an electric current. A technique for the fractionation of magnetic fluids has been developed. Two different magnetic fluids and different batches of one magnetic fluid have been fractionated by this method. The fractions yielded are 
characterized with respect to their size and magnetic properties. Some of the magnetic characterization methods are related to biomedical applications of magnetic fluids.

\section{BIOMEDICAL APPLICATIONS OF MAGNETIC NANOPARTICLES}

Magnetic fluids or nanoparticles applied in biomedicine are mainly based on water as a carrier liquid. There are several well established applications, whereas others are still under development. Examples of both are given in the following.

\section{Biological Separation}

Different methods are applied to the separation of biological substances like microorganisms, cells, nucleic acids or proteins from complex suspensions such as blood. Examples are immunocolumns containing antibodies bound to the column material, centrifugation (often performed with density gradient) and cell sorting by flow cytometry, which separates single cells in accordance with their optical characteristics. Furthermore, magnetic particles coupled, for example, to antibodies are widely used. Biological substances labeled with magnetic particles can be isolated and enriched by magnetic fields to a high purity within minutes. Both labeled and non-labeled fractions can be completely recovered. Magnetic polymer particles in the micrometer range are often used for such purposes [7]. They can be separated by a simple magnet, but relatively large particles settle quickly. Magnetic nanoparticles are also applied as an alternative to such large particles [8-10]. They are stable in liquids, but biological substances labeled with these particles require the high-gradient magnetic separation, e.g., columns filled with soft magnetic iron spheres, steel wool or steel nets.

\section{Magnetic Resonance Imaging}

Magnetic resonance imaging or tomography is an important technique in medical diagnostics using nuclear magnetic resonance [11]. For 
imaging purposes, besides the density of the nuclear spins of common protons, the longitudinal relaxation time $T_{1}$ and the transverse relaxation time $T_{2}$ of the spins are used. The presence of magnetic substances in a volume under investigation alters the local magnetic field due to their susceptibility, resulting in shorter relaxation times. This effect increases with the concentration of the magnetic substance. Plots of the reciprocal relaxation times as a function of the concentration yield straight lines. Their slopes are called relaxivity $r 1$ for the longitudinal relaxation and relaxivity $r 2$ for the transverse relaxation.

Hence, in the magnetic resonance imaging biocompatible magnetic substances are used as contrast agents. These substances are, for example, solutions of paramagnetic substances or magnetic fluids which are injected or orally administered. Nanoparticles like those contained in magnetic fluids are predominantly cleared from the blood by the liver, spleen and bone marrow. The clearance rate depends on various particle characteristics. Therefore, magnetic fluids are applied as contrast agents for magnetic resonance imaging of, for example, the liver, blood pool or gastrointestinal tract [12]. They especially shorten the transverse relaxation time $T_{2}$.

\section{Magnetorelaxometry}

Immunoassays are commonly applied to the quantification of biologically active substances like antibodies or antigens. For that purpose the reaction partners are labeled with signal generators like fluorescent dyes, radionuclides or enzymes. Recently a method for the evaluation of immunoassays was introduced, the so-called magnetorelaxometry, which uses magnetic nanoparticles as signal generators [13]. This method observes the relaxation of the net magnetic moment of a system of magnetic nanoparticles after the removal of a magnetic field, also known as magnetic viscosity. Magnetorelaxometry utilizes the fact that after the removal of an external magnetic field there are two different relaxation mechanisms. On the one hand, particles accomplish rotational diffusion within a carrier liquid, which is called Brownian relaxation [14]. The corresponding relaxation time is

$$
\tau_{\text {Brown }}=\frac{4 \pi r^{3} \eta}{k T}
$$


for spherical particles with the hydrodynamic radius $r$, where $\eta$ is the dynamic viscosity of the carrier liquid, $k$ the Boltzmann constant and $T$ the absolute temperature.

On the other hand, the Neel relaxation describes the rotation of the magnetization vector inside the magnetic core against an energy barrier [15]. The energy barrier is given by the product of the volume of the magnetic core $V_{\text {core }}$ and the magnetic anisotropy constant $K$, which is mainly caused by the crystalline and shape anisotropy. The relaxation time of this process is given by

$$
\tau_{\text {Néel }}=\tau_{0} \exp \left(\frac{K V_{\text {core }}}{k T}\right)
$$

where $\tau_{0}$ is usually approximated as $10^{-9} \mathrm{~s}$. For systems of nanoparticles suspended in liquids the faster relaxation mechanism dominates the relaxation process. If magnetic nanoparticles are immobilized, magnetic relaxation is only possible in accordance with the Néel relaxation.

For an ensemble of identical particles the reduction of the magnetization $M$ as a function of time $t$ after the removal of a weak magnetic field of the strength $H_{0}$ is described for both relaxation processes by a single exponential decay with the appropriate time constant $\tau$

$$
M(t)=M_{0} \exp (-t / \tau) \sim \frac{n m^{2} H_{0}}{k T} \exp (-t / \tau)
$$

where $n$ is the number of particles with the magnetic moment $m$, which is the product of the core volume and the saturation magnetization. Therefore, the amplitude of the relaxation signal depends on the square of the magnetic moment of the particles.

However. real magnetic fluids contain nanoparticles with different hydrodynamic sizes and thus different relaxation times. Therefore, for Brownian relaxation the time dependence of the magnetization can be described as a superposition of exponential decays.

$$
M_{\text {Brown }}(t)=\int_{0}^{\infty} M_{0, \text { Brown }}\left(\tau_{\text {Brown }}\right) \exp \left(-t / \tau_{\text {Brown }}\right) d \tau_{\text {Brown }}
$$

A typical relaxation signal from a liquid sample is shown in Figure 1. From this approximately exponential decay relaxation times can be 


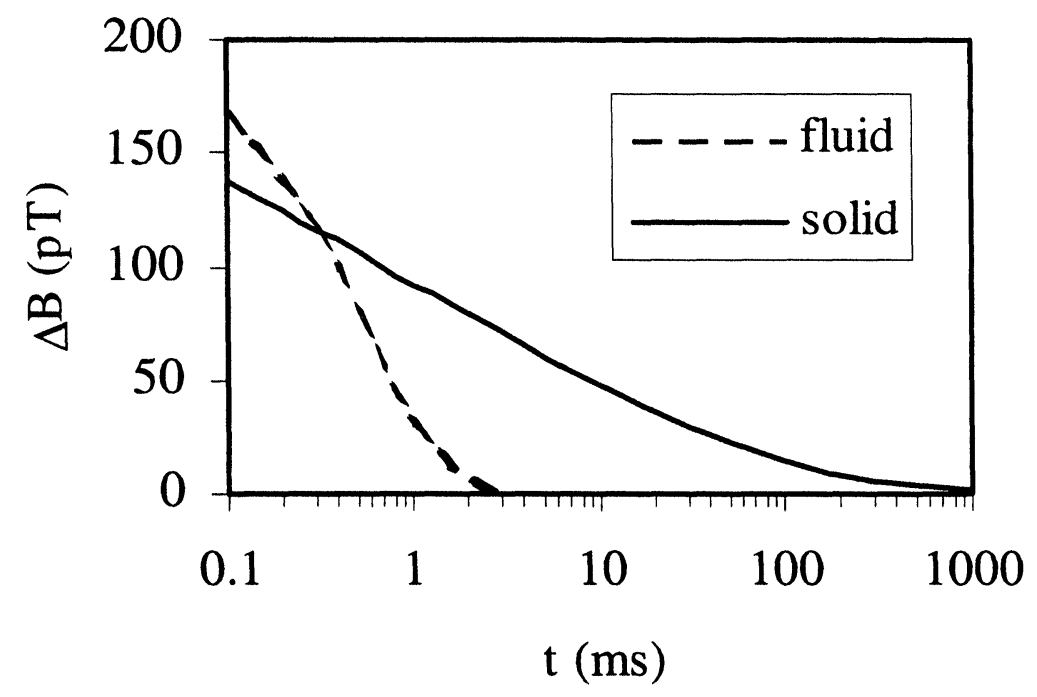

FIGURE 1 Relaxation of the net magnetic moment of a liquid and a freeze-dried sample of magnetic nanoparticles after the removal of a magnetic field.

estimated. Furthermore, real magnetic nanoparticle systems show a distribution of core size and magnetic anisotropy. The Néel relaxation time depends exponentially on these two dispersed quantities, which lead to a logarithmic decrease of the magnetization as a function of time [16]

$$
M_{\text {Néel }}(t)=M_{0, \text { Néel }} \ln \left(1+t_{c} / t\right)
$$

where the characteristic time $t_{c}$ depends on the magnetization time and field. These two times are equal for weak magnetizing fields [17]. Figure 1 also contains a typical relaxation signal of immobilized nanoparticles. The Néel and Brownian relaxation can be distinguished as result of their different time dependences, which in turn makes it possible to discriminate between free antibodies labeled with magnetic nanoparticles and those which have bound to the reaction partner [18]. Therefore, a previous separation step can be avoided in magnetorelaxometry.

In order to detect the Brownian relaxation magnetically, the magnetic core must be stable (remnant) during the measurement. However, for the detection of a Néel relaxation signal the relaxation 
times of the magnetic cores must be within the observed time window. Magnetic cores with relaxation times that are suitable for such measurements represent only a small portion of the common core size and anisotropy distribution. Larger magnetic cores are usually remnant and thus suitable for the determination of Brownian relaxation signals. Hence, magnetic nanoparticles, at least part of them, must fulfill several requirements for application in magnetorelaxometry.

\section{Hyperthermia}

A local heating of certain organs or tissues to temperatures between 314 and $320 \mathrm{~K}$, especially for cancer therapy, is called hyperthermia [19]. This heating can be achieved by means of external sources such as radio frequencies, microwaves, infrared or water baths. Furthermore, magnetically induced heating has been tested using external alternating magnetic fields to inductively heat magnetic materials that have been introduced into the tumor. For that purpose magnetic seeds must be implanted, whereas magnetic fluids can be injected.

In alternating magnetic fields multidomain materials show hysteretic and Rayleigh losses [20]. In the case of electrically conductive materials there is an additional contribution by eddy currents. Its power increases with the square of the frequency. For single domain nanoparticles the loss mechanisms are the Brownian and Néel relaxation mentioned above. For both mechanisms the specific absorption rates or power losses $P$ are

$$
P \sim \frac{2 \pi f \mu_{0} H^{2}}{2} \frac{\mu_{0} n m^{2}}{k T} \frac{2 \pi f \tau}{1+(2 \pi f \tau)^{2}}
$$

where $f$ is the frequency and $\mu_{0}$ the magnetic permeability of the vacuum.

The dependence of the loss on the various parameters mentioned can be gathered from Eq. (6). The loss as a function of the frequency corresponds to the imaginary part of the alternating or complex magnetic susceptibility. This imaginary part has maxima at the so-called resonant frequencies. Susceptometry measures the magnetic response as a function of the frequency and is related to 
magnetorelaxometry that observes the magnetic response as a function of time. Hence, resonant frequencies are related to the reciprocals of the corresponding relaxation times. For common magnetic fluids the resonant frequency of the Brownian relaxation is below $100 \mathrm{kHz}$ [21], whereas the one based on Néel relaxation is in the $\mathrm{GHz}$ range [22].

However, the Néel relaxation in particular covers a wide frequency range due to the distribution of different parameters, as mentioned above. In normal tissues magnetic fields with frequencies greater than several $\mathrm{MHz}$ generate significant heat due to eddy currents resulting from ionic conductivity [23]. Furthermore, at frequencies below a few $\mathrm{kHz}$ the power loss is low and the induced currents can irritate nerves and muscles. Therefore, magnetic fields with intermediate frequencies are preferred. Additionally, the maximum magnetic field strength is limited by the tissue tolerance. Since the magnetic fluid hyperthermia has been used successfully for the treatment of cancer cells and tumors in animals, clinical trials on brain tumors are planned [24].

\section{MATERIALS AND METHODS}

Two different samples of common aqueous magnetic fluids based on polymer-stabilized iron-oxide nanoparticles, mainly maghemite, were studied. In magnetic fluid 1 the particles were coated with carbohydrate dextran. They were produced by a single-step synthesis [25]. The saturation magnetization was about $4.5 \mathrm{Am}^{2} \mathrm{~mol}^{-1} \mathrm{Fe}$. The particles of magnetic fluid 2 were stabilized with polyethylene glycol in a second step after core synthesis [26]. The saturation magnetization was about $6 \mathrm{Am}^{2} \mathrm{Fe}$. Unless otherwise noted, fractionations and measurements were made at room temperature.

A method for magnetic fractionation of magnetic fluids has been developed. The set-up consisted of an electromagnet with variable pole shoes and a variable power supply (Oxford Instruments, Oxford, UK). It generates a maximum magnetic flux density of $1 \mathrm{~T}$ in a $10 \mathrm{~mm}$ air gap. Since the retention of magnetic nanoparticles requires high magnetic field gradients, a column of about $23 \mathrm{~mm}$ inner diameter and $40 \mathrm{~mm}$ length filled with soft magnetic iron spheres of $0.3 \mathrm{~mm}$ diameter (Miltenyi Biotech, Bergisch Gladbach, Germany) was placed between the pole shoes. 
For fractionation a magnetic fluid was poured into the column with the highest magnetic field strength applied. The column was then washed with approximately $500 \mathrm{~mm}^{3} \mathrm{~s}^{-1}$ of deionized water until the collected effluent was almost colorless, i.e., it contained only a few magnetic particles. The current, and thus the magnetic field, was then gradually reduced. The columns were washed again with deionized water until the effluent was colorless. This procedure had been repeated stepwise until the field was decreased to zero.

The hydrodynamic size of magnetic nanoparticles was measured at $298 \mathrm{~K}$ by photon correlation spectroscopy using a Zetasizer 3000 (Malvern Instruments, Malvern, UK) with a He Ne Laser at $633 \mathrm{~nm}$. The results are given as the so-called $\mathrm{Z}$-averages and polydispersities, which are measures of the width of the distribution, of the hydrodynamic size.

The iron content was measured using an optical emission spectroscope ICP-OES 3560 B Analyzer (ARL, Offenbach, Germany). Magnetization curves up to $740 \mathrm{kA} \mathrm{m}^{-1}$ were measured at the LI2CEquipe Colloïdes Magnétique (University of Paris 6) using a homemade vibrating sample magnetometer according to Foner.

The influence of magnetic fluids on the nuclear magnetic resonance of water protons was recorded with a minispec pc 120 (Bruker, Karlsruhe, Germany) at $0.47 \mathrm{~T}(20 \mathrm{MHz})$ and $313 \mathrm{~K}$. The inversion recovery method was used for measurements of the longitudinal relaxation time, the Carr-Purcell-Meiboom-Gill sequence for the determination of the transverse relaxation time.

For magnetic relaxation measurements an electronic gradiometer consisting of two DC-SQUID (Superconducting Quantum Interference Device) magnetometers was employed. It was developed by the Physikalisch-Technische Bundesanstalt [27]. The decay of the magnetic flux density of the samples $\left(\leq 150 \mathrm{~mm}^{3},<1 \mathrm{~mol} \mathrm{Fe} \mathrm{m}^{-3}\right)$ was recorded after magnetization at a maximum of $1.2 \mathrm{kA} \mathrm{m}^{-1}$ for $1.15 \mathrm{~s}$. In the case of freeze-dried samples, the difference in the magnetic flux density was determined between 0.6 to $480.6 \mathrm{~ms}$. It is called the Néel relaxation amplitude in keeping with the underlying relaxation mechanism. For liquid samples, the difference between 0.6 and $80.6 \mathrm{~ms}$ was calculated and is referred to as the Brownian relaxation amplitude. Furthermore, the time constant after which the magnetic flux density had decreased to $e^{-1}$ of the calculated Brownian 
relaxation amplitude was determined. This time constant is an estimate of the Brownian relaxation time. Thus, the hydrodynamic size can be calculated according to Eq. (1).

\section{RESULTS}

First, magnetic fluid 1 was fractionated by the magnetic method. The fractions were collected at stepwise decreasing magnetic flux densities from 1 down to $0 \mathrm{~T}$. The first effluent at the highest magnetic flux density was collected in the first fraction $(1000 \mathrm{mT} / 1)$, whereas the larger remainder went into the second fraction $(1000 \mathrm{mT} / 2)$. Further investigations showed that the retention of magnetic particles increases with a decreasing flow rate.

The saturation magnetization was determined from magnetization curves, which could be evaluated with the exception of the fraction collected at the lowest magnetic flux density, whereas the iron concentration was determined by optical emission spectroscopy. Figure 2 shows the iron amount, the product of the concentration and the volume of a sample, and the saturation magnetization of magnetic fluid

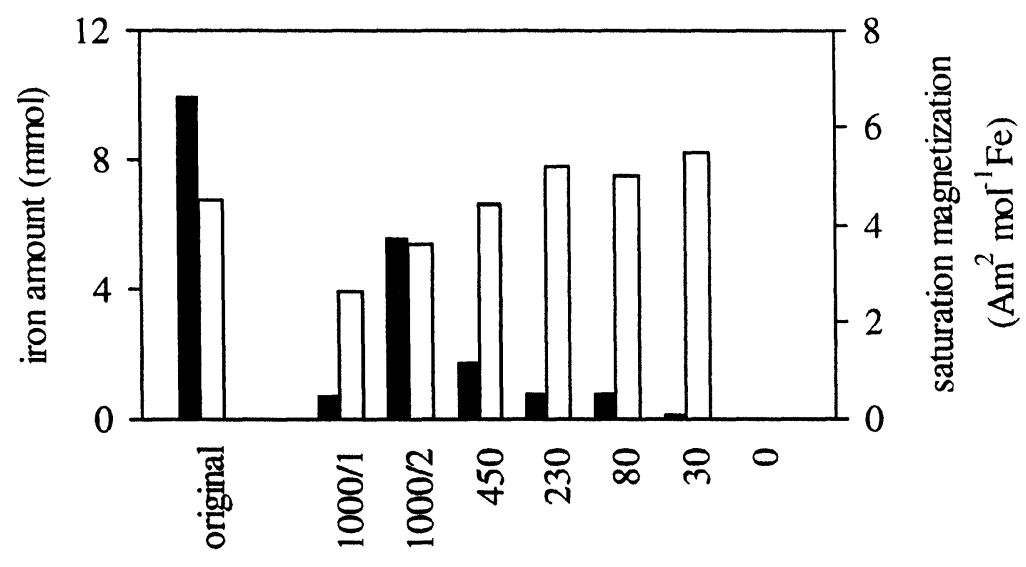

\section{fraction $(\mathrm{mT})$}

FIGURE 2 Magnetic fractionation of magnetic fluid 1: iron content (closed bar) and saturation magnetization (open bar) of the original sample and the fractions designated in accordance with the releasing magnetic flux density. 
1 and its fractions. More than half of the total iron amount is in the second fraction collected at the highest magnetic flux density. The saturation magnetizations of the fractions, which increase with a decreasing magnetic field, are approximately that of the original sample.

The magnetic core size can be estimated from magnetization curves. For evaluation a model is used that assumes a lognormal size distribution [28]. Figure 3 shows the average core size and its standard deviation of magnetic fluid 1 and its magnetic fractions. The core size of the fractions increases from below to above the size of the original sample with the decreasing magnetic field. The standard deviations of all fractions tend to be smaller than that of the original sample.

The hydrodynamic size and the polydispersity of magnetic fluid 1 and its magnetic fractions obtained by photon correlation spectroscopy are shown in Figure 4. The hydrodynamic size of fractions increases with the decreasing magnetic field and covers a wide range around that of the original sample. The polydispersities of the fractions are somewhat lower than that of the original sample.

For magnetic fluid hyperthermia, Figure 5 shows the specific absorption rate of the liquid samples in a magnetic field of $12.5 \mathrm{kA} \mathrm{m}^{-1}$

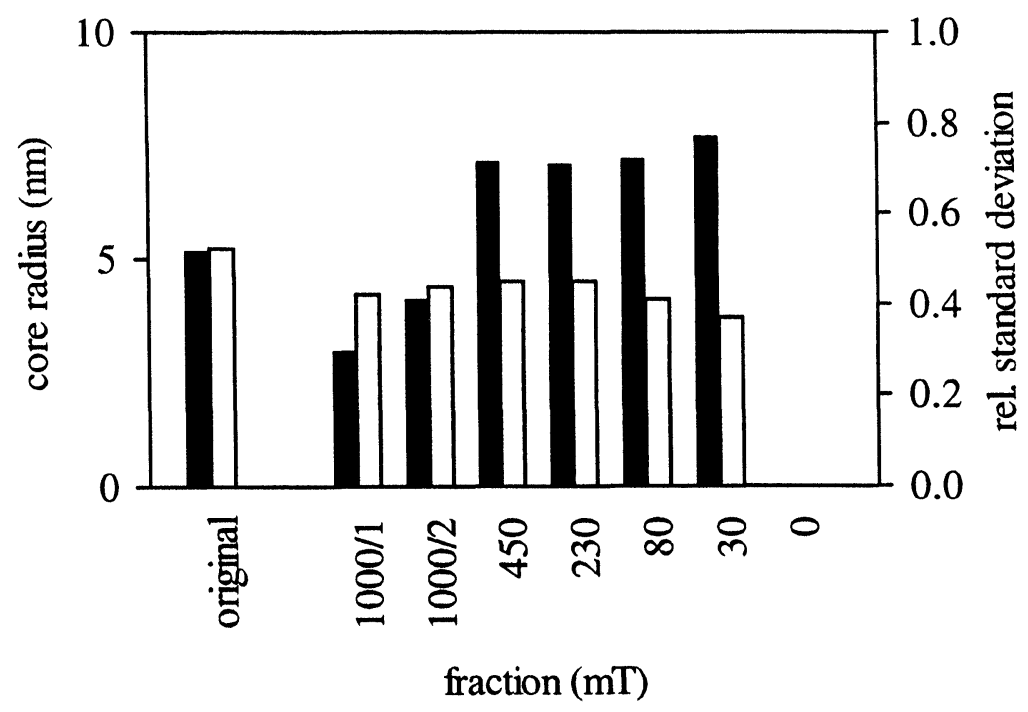

FIGURE 3 The core radius (closed bar) and its standard deviation (open bar), both calculated from magnetization curves, of magnetic fluid 1 and its magnetic fractions. 


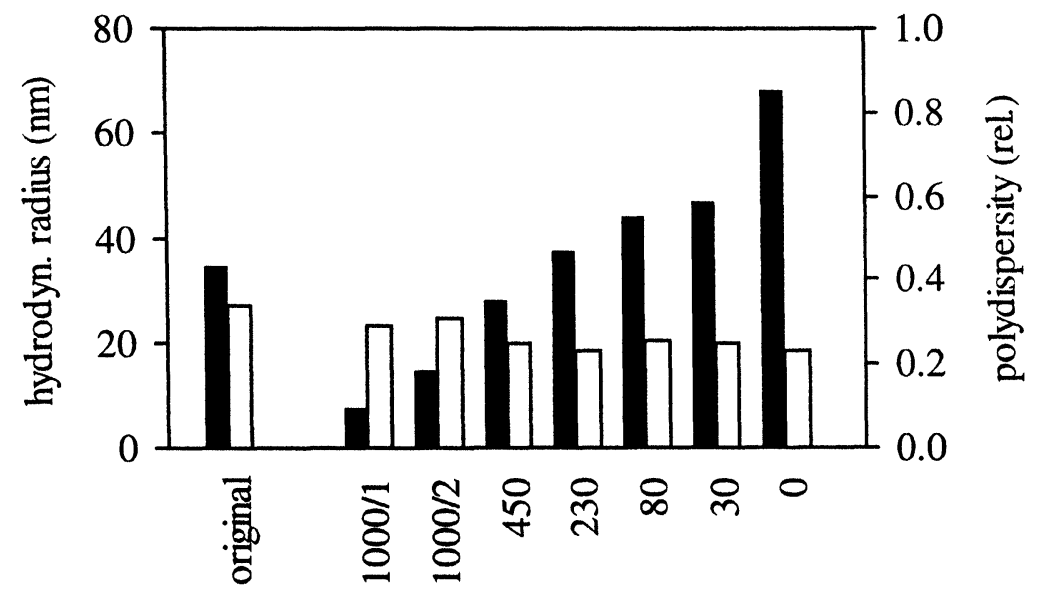

fraction $(\mathrm{mT})$

FIGURE 4 Photon correlation spectroscopy: hydrodynamic radius (closed bar) and polydispersity (open bar) of magnetic fluid 1 and its magnetic fractions.

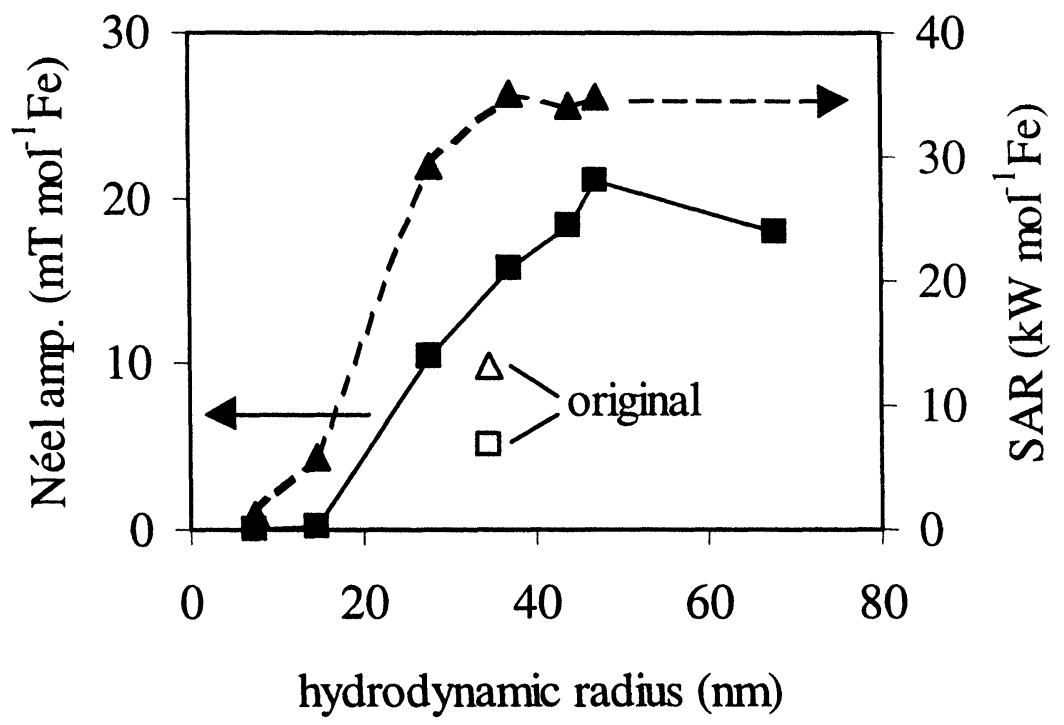

FIGURE 5 Solid phase magnetorelaxometry and magnetic fluid hyperthermia: Néel relaxation amplitude and specific absorption rate of magnetic fluid 1 and its magnetic fractions as a function of the hydrodynamic radius. 
and $0.5 \mathrm{MHz}$ [29]. Specific absorption rates of fractions increase with the hydrodynamic size and are distinctly different from the original sample.

Magnetic relaxation investigations of dilution series involving immobilized as well as liquid magnetic fluids showed that the relaxation amplitudes are directly proportional to the iron content of the samples [30]. Therefore, in the following the Néel and Brownian relaxation amplitude are related to the iron content in order to compare different samples. The Néel relaxation amplitude of freezedried samples of magnetic fluid 1 and its magnetic fractions is plotted in Figure 5 as a function of the hydrodynamic size. The Néel relaxation amplitudes of fractions increase with the hydrodynamic size and clearly differ from the original sample.

For the corresponding liquid samples the Brownian relaxation amplitude and the hydrodynamic size, calculated from the Brownian relaxation time, are shown in Figure 6 as a function of the hydrodynamic size measured by photon correlation spectroscopy. The behavior of the Brownian relaxation amplitudes of the fractions with

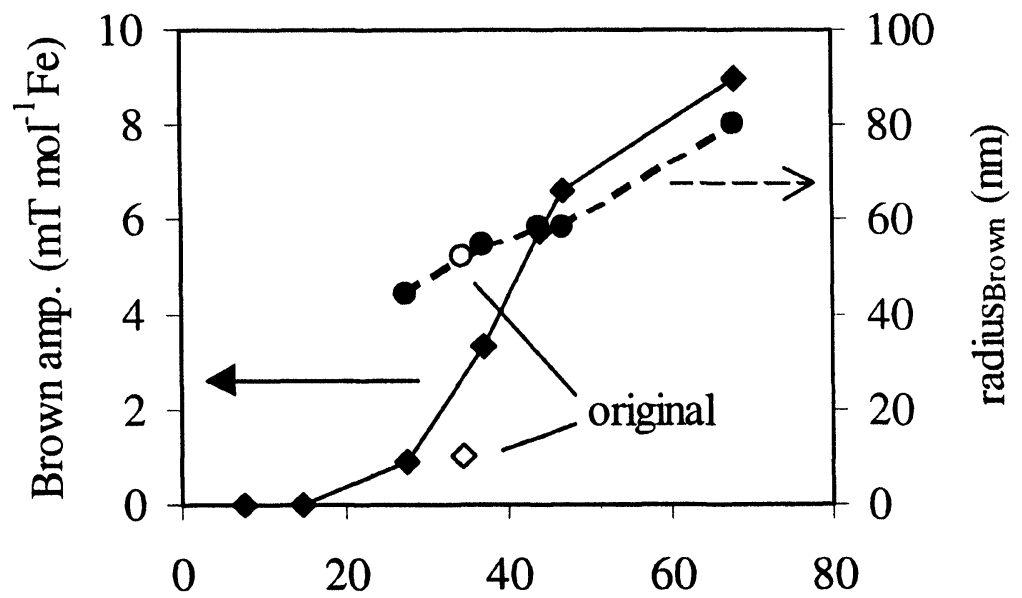

hydrodynamic radius $\mathrm{PCS}(\mathrm{nm})$

FIGURE 6 Liquid phase magnetorelaxometry: Brownian relaxation amplitude and hydrodynamic radius, calculated from the Brownian relaxation time, of magnetic fluid 1 and its magnetic fractions as a function of the hydrodynamic radius measured by photon correlation spectroscopy (PCS). 
increasing hydrodynamic size resembles that of the Néel relaxation amplitudes. The hydrodynamic sizes measured by magnetorelaxometry are generally higher than the values obtained by photon correlation spectroscopy, but the increases in size are quite comparable.

Magnetic resonance was used for characterization of the samples, too. Figure 7 shows the relaxivity $r 2$ of two different batches of magnetic fluid 1 and their magnetic fractions as a function of the hydrodynamic size. The relaxivity $r 2$ of fractions increases with the hydrodynamic size. Relaxivities $r 2$ of fractions cover a relatively broad range and are distinctly different from the original samples. The relaxivity $r 1$ is not plotted because relaxivities $r 1$ of the original samples and the magnetic fractions, with the exception of the first fraction, are nearly identical. Although the original samples of the two batches differ in hydrodynamic size and relaxivity $r 2$, relaxivities $r 2$ of the fractions as a function of the hydrodynamic size correlate well.

Magnetic fluid 2 was fractionated to check whether magnetic fractionation also works with other magnetic fluids. The fractions yielded and the original sample were investigated. It is found that the hydrodynamic size of the fractions increases with a decreasing magnetic field. Figure 8 shows the Néel relaxation amplitude and

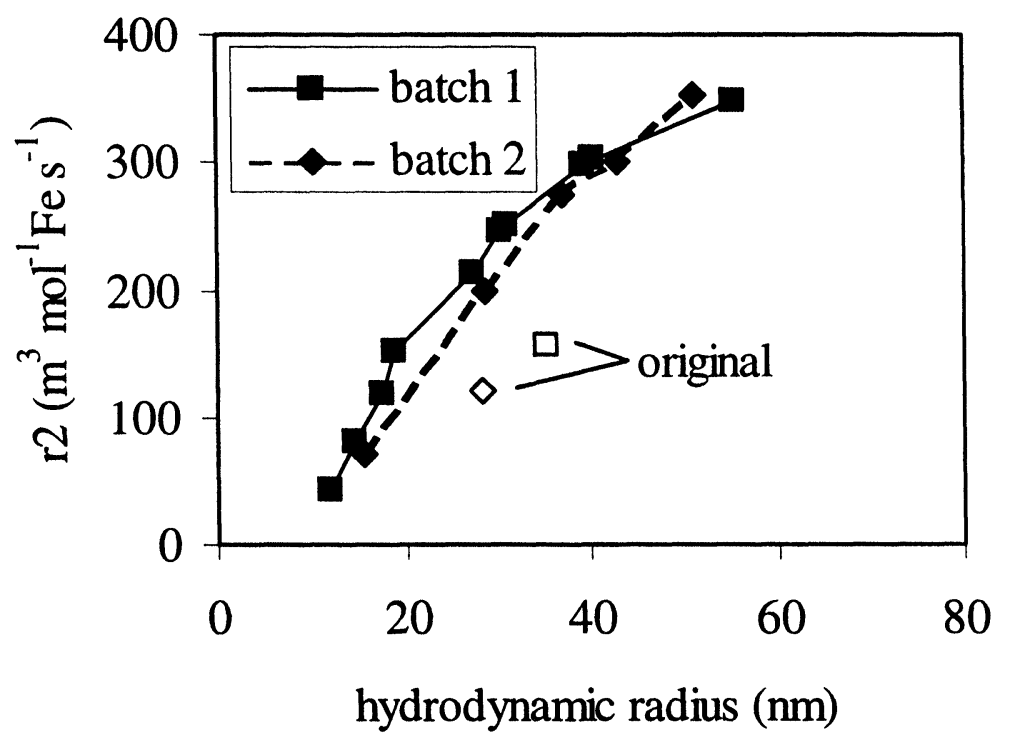

FIGURE 7 Magnetic resonance: relaxivity $r 2$ of two batches of magnetic fluid 1 and their magnetic fractions as a function of the hydrodynamic radius. 


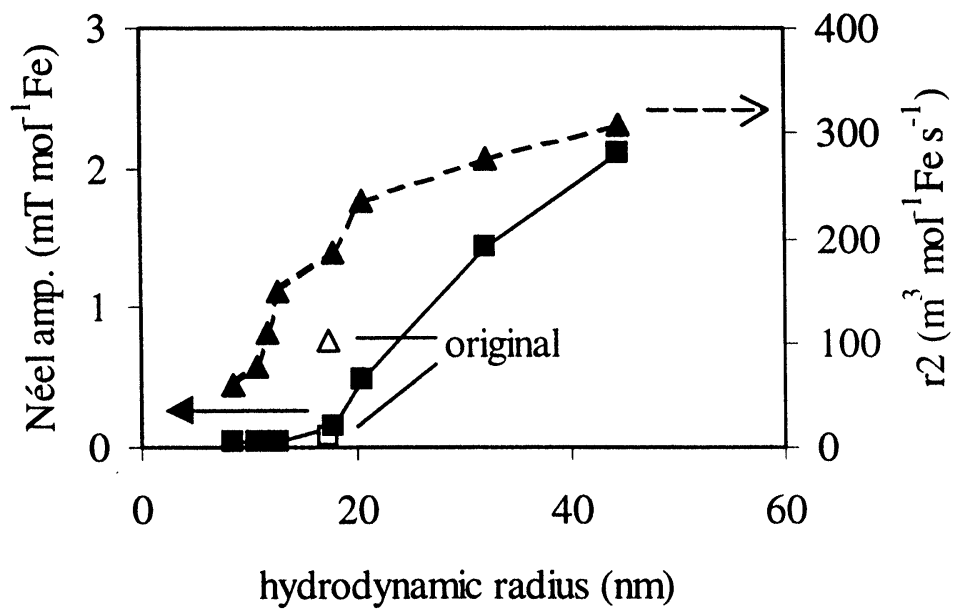

FIGURE 8 Magnetic fractionation of magnetic fluid 2: Néel relaxation amplitude and relaxivity $r 2$ of the original sample and its magnetic fractions as a function of the hydrodynamic radius.

relaxivity $r 2$ of the original sample and its fractions as a function of the hydrodynamic size. In the case of the fractions both magnetic quantities increase with the hydrodynamic size. The Néel relaxation amplitude, relaxivity $r 2$ and hydrodynamic size each cover a broad range and in part clearly differ from those of the original sample.

\section{DISCUSSION}

The results show that magnetic fractionation divides magnetic fluids into several fractions with different magnetic and non-magnetic properties. In the case of magnetic fluid 1, Figure 2 shows that more than half of the magnetic nanoparticles by the iron amount cannot be held back despite the high maximum flux density, high field gradients and the reduced flow rate. Furthermore, the amount of iron on the column decreases with the magnetic flux density. Moreover, the core size of the fractions, which is proportional to the magnetic moment, increases with the decreasing magnetic field, as can be seen in Figure 3. This indicates that magnetic fractionation indeed separates nanoparticles in accordance with their magnetic moment. The standard deviation of the original sample is slightly higher than that of the fractions. Hence, magnetic fractionation mainly shifts the mean core size 
and reduces the standard deviation only gradually. Figure 2 further shows that the specific saturation magnetization of fractions increases with the decreasing magnetic field and thus correlates with the core size. This correlation can be explained by the fact that the relative portion of the non-magnetic surface region is reduced with the increasing core size. Fractions collected at magnetic flux densities lower than $1 \mathrm{~T}$ have the saturation magnetization close to $6.4 \mathrm{Am}^{2} \mathrm{~mol}^{-1} \mathrm{Fe}$ for bulk maghemite, the main core material [31]. Therefore, the major portion of these cores is ferrimagnetic despite the nanoscale.

The hydrodynamic size of fractions of both magnetic fluids increases with the decreasing magnetic field. This indicates that the hydrodynamic size correlates with the core size for the investigated magnetic fluids. For magnetic fluid 1, Figures 2 and 4 make it evident that the majority of particles by weight have the hydrodynamic radius of less than $20 \mathrm{~nm}$. Furthermore, the polydispersities of fractions are somewhat lower than that of the original sample. Therefore, magnetic fractionation mainly shifts the mean hydrodynamic size and reduces the polydispersity only slightly. This is similar to the results obtained for the core size. However, hydrodynamic sizes of magnetic nanoparticles are much greater than the core sizes. One reason for this is that for the polymer-coated nanoparticles investigated the hydrodynamic size is greater than the core size. Additionally, in the case of polydispersed systems the intensity-weighted mean from photon correlation spectroscopy is larger than the volume-weighted one from magnetization curves.

The Néel relaxation amplitude of fractions of both magnetic fluids increases with the hydrodynamic size, as Figures 5 and 8 show. Taking into account the results given in Figure 4, the Néel relaxation amplitude of the fractions of magnetic fluid 1 increases with the decreasing magnetic field, too. This is also valid for magnetic fluid 2 . Furthermore, Figure 6 shows for magnetic fluid 1 that the Brownian relaxation amplitude of fractions greatly increases with the hydrodynamic size and thus with the decreasing magnetic field.

Both, the Néel and Brownian relaxation amplitudes, are measures of the number of magnetic cores that magnetorelaxometry can detect. For Néel relaxation, the cores must have Néel relaxation times within the observed time window, whereas larger magnetically remnant cores are required for Brownian relaxation. These magnetic cores are 
relatively large for low anisotropic magnetic materials like the ironoxide used. They represent only small parts of the core distribution. These parts and thus the relaxation amplitudes distinguish between different magnetic fluids. In the case of fractions of a magnetic fluid, the increasing relaxation amplitudes correspond to the increasing mean core size, as found above. For fractions collected at the highest magnetic flux density neither Néel nor Brownian relaxation signals could be detected. These fractions contain particles with small core and hydrodynamic sizes, as mentioned above. Therefore, they do not yield a relaxation signal in the observed time window. Furthermore, the hydrodynamic sizes calculated from the Brownian relaxation times are larger than those obtained by photon correlation spectroscopy. This is mainly due to the fact that the relaxation processes much faster than the delay time of $0.6 \mathrm{~ms}$ are practically neglected. Additionally, magnetorelaxometry can record only nanoparticles with magnetically remnant cores, whereas photon correlation spectroscopy responds to all nanoparticles.

In the magnetic fluid hyperthermia the specific absorption rate of the fractions of magnetic fluid 1 increases with the hydrodynamic size, as Figure 5 shows. Taking into account the results given in Figures 3 and 4 , the specific absorption rate increases with the core size. This finding is in accordance with the theory in Eq. (6).

Figures 7 and 8 show that the relaxivity $r 2$ of fractions of both magnetic fluids increases with the hydrodynamic size and with the decreasing magnetic field. It is known that the relaxivity $r 2$ increases with the magnetic core size of single-domain particles [32]. This corresponds to the core sizes found for the fractions.

Several magnetic fractionations even of different batches of magnetic fluid 1 display good reproducibility, as Figure 7 shows. Although the batches differ in hydrodynamic size and relaxivity $r 2$, fractions with a similar hydrodynamic size have similar relaxivities $r 2$. Furthermore, two different magnetic fluids with different synthesis and coating material have been successfully fractionated by the magnetic method. Therefore, the results do not seem to be restricted to the magnetic fluids described but appear to be more generally valid.

For both magnetic fluids the hydrodynamic size, Néel relaxation amplitude and relaxivity $r 2$ of fractions cover relatively broad ranges, which imply a broad distribution of these parameters in the original 
samples. The specific Néel relaxation amplitude, which is important in solid phase assays for magnetorelaxometry, can be increased more than tenfold in the case of magnetic fluid 2, as Figure 8 shows. The improvement in the Brownian relaxation amplitude that is relevant for liquid phase assays is mostly larger than that of the Néel relaxation amplitude (compare Figs. 5 and 6). Furthermore, the specific absorption rate of fractions containing large particles is three times higher than that of the original sample, as can be seen in Figure 5. A similar enhancement is found for the relaxivity $r 2$ according to Figures 7 and 8 . Therefore, fractions containing large particles are especially suitable for applications like magnetic resonance, magnetorelaxometry or magnetic fluid hyperthermia.

The retention of magnetic particles by magnetic fractionation increases with the magnetic flux density and core size, as. Figures 2 and 3 show. Furthermore, magnetic nanoparticles cannot be held back without high magnetic field gradients through steel wool, small spheres, nets etc. The flow rate also has an influence, as mentioned. Therefore, the retention of particles increases with the magnetic moment of the nanoparticle, the applied magnetic field and field gradient, and with the decreasing flow rate. The same dependences have been found for high-gradient magnetic separation of submicron particles [6]. Hence, there is a competition between magnetic and hydrodynamic forces. The situation is very complex because the core and shell of magnetic nanoparticles have different compositions and characteristics. Thus, magnetic interactions depend on small cores, whereas the hydrodynamic interactions are influenced by the larger polymer coating. Furthermore, both core and hydrodynamic size are widely distributed and need not be correlated.

\section{SUMMARY}

There are various biomedical applications for magnetic fluids. Thus, magnetic particles are commercially available for the biological separation of cells, nucleic acids etc., or as contrast agents for magnetic resonance imaging, an important method in medical diagnostics. A method for the quantification of biologically active substances that uses magnetic nanoparticles as markers was recently 
suggested. Finally, magnetic fluids are being tested for cancer therapy based on inductive heating in alternating magnetic fields.

A simple magnetic method for the fractionation of magnetic fluids is presented. The method is a high-gradient magnetic separation of magnetic nanoparticles. It was shown that this technique separates magnetic nanoparticles according to their magnetic moments, which are utilized in the applications of magnetic fluids. Furthermore, the method fractionates different magnetic fluids successfully and has a good reproducibility. Magnetic properties of fractions yielded clearly differ from those of the original samples. Therefore, particles obtained in this way are better suited for a number of applications. Hence, magnetic fractionation can optimize considerably magnetic fluids with respect to their applications.

\section{Acknowledgments}

The financial support of the BMBF by grant 13 N7005/1 is acknowledged. We would also like to thank the Physikalisch-Technische Bundesanstalt Berlin for the opportunity to perform the magnetorelaxometry measurements. Furthermore, we wish to thank the LI2CEquipe Colloïdes Magnétique of the University of Paris 6 for making the vibrating sample magnetometer available to us.

\section{References}

[1] Raj, K. and Moskowitz, R. (1990). J. Magn. Magn. Mater., 85, 233.

[2] Sjögren, C. E., Johansson, C., Nævestad, A., Sontum, P. C., Briley-Sæbö, K. and Fahlvik, A. K. (1997). Magn. Res. Imag., 15, 55.

[3] Nunes, A. C. and Yu, Z. C. (1989). J. Magn. Magn. Mater., 78, 241.

[4] O'Grady, K., Stewardson, H. R., Chantrell, R. W., Fletcher, D., Unwin, D. and Parker, M. R. (1986). IEEE Trans. Magn., 22, 1134.

[5] Kirpotin, D., Chan, D. C. F. and Bunn, P. A. (1993). US 5411730.

[6] Takayasu, M., Gerber, R. and Friedlaender, F. J. (1983). IEEE Trans. Magn., 19, 2112.

[7] Luxembourg, A. T., Borrow, P., Teyton, L., Brunmark, A. B., Peterson, P. A. and Jackson, M. R. (1998). Nature Biotech., 16, 281.

[8] Miltenyi, S., Müller, W., Weichel, W. and Radbruch, A. (1990). Cytometry, 11, 231.

[9] Thomas, T. E., Abraham, S. J. R., Philips, G. L. and Lansdorp, P. M. (1994). Blood, 84, 101a.

[10] Kemshead, J. T., Hancock, J. and Liberti, P. (1994). J. Hematotherapy, 3, 51.

[11] Reiser, M. and Semmler, W. (Eds.), Magnetresonanztomographie, 2nd edn., Springer, Berlin, 1997. 
[12] Frija, G., Clément, O. and de Kerviler, E. (1994). Invest. Radiol., 29, S75.

[13] Weitschies, W., Kötitz, R., Bunte, T. and Trahms, L. (1997). Pharm. Pharmacol. Letter, $7,5$.

[14] Debye, P., Polar Molecules, Chemical Catalog Company, New York, 1929.

[15] Néel, L. (1949). Ann. Geophys., 5, 99.

[16] Chantrell, R. W., Hoon, S. R. and Tanner, B. K. (1983). J. Magn. Magn. Mater., 38, 133.

[17] Berkov, D. V. and Kötitz, R. (1996). J. Phys.: Condens. Matter, 8, 1257.

[18] Kötitz, R., Weitschies, W., Trahms, L., Brewer, W. and Semmler, W. (1999). J. Magn. Magn. Mater., 194, 62.

[19] Jordan, A., Scholz, R., Wust, P., Fähling, H. and Felix, R. (1999). J. Magn. Magn. Mater., 201, 413.

[20] Hiergeist, R., Andrä, W., Buske, N., Hergt, R., Hilger, I., Richter, U. and Kaiser, W. (1999). J. Magn. Magn. Mater., 201, 420.

[21] Rheinländer, T., Justiz, J., Haller, A., Kötitz, R., Weitschies, W. and Semmler, W. (1999). IEEE Trans. Magn., 34, 4055.

[22] Fannin, P. C., Relihan, T. and Charles, S. W. (1995). J. Phys. D: Appl. Phy., 28, 2003.

[23] Borrelli, N. F., Luderer, A. A., Mansfield, G. R. and Panzarino, J. N. (1983). US 4574782.

[24] Jentsch, G. (2000). Focus, 10/2000, 180.

[25] Hasegawa, M. (1987). US 4101435.

[26] Pilgrimm, H. (1992). US 5160725.

[27] Matz, H., Drung, D., Hartwig, S., Groß, H., Kötitz, R., Müller, W., Vass, A., Weitschies, W. and Trahms, L. (1998). Appl. Supercond., 6, 557.

[28] Chantrell, R. W., Popplewell, J. and Charles, S. W. (1978). IEEE Trans. Magn., 14, 975.

[29] Jordan, A. (1998). personal communication.

[30] Haller, A., Hartwig, S., Matz, H., Lange, J., Rheinländer, T., Kötitz, R., Weitschies, W. and Trahms, L. (1999). Supercond. Sci. Techn., 12, 956.

[31] Craik, D., Magnetism, Wiley, Chichester, UK, 1995, p. 379.

[32] Muller, R. N., Gillis, P., Moiny, F. and Roch, A. (1991). Magn. Res. Med., 22, 178. 


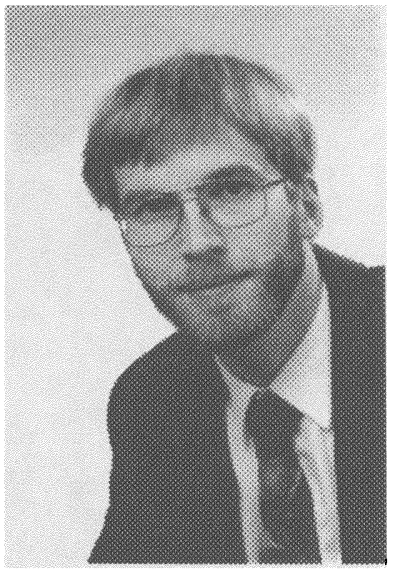

T.Rheinländer has been involved in physical and colloidal chemistry since his diploma thesis. After he had obtained his doctorate at the Research Centre Jülich he worked as a post-doctoral fellow at the Max Planck Institute of Colloids and Interfaces in Berlin. There he developed methods for the characterisation and optimisation of magnetic fluids. In April 2000 he was appointed a project leader at Mifa AG, Switzerland. 